\title{
Intracameral cefuroxime and moxifloxacin used as endophthalmitis prophylaxis after cataract surgery: systematic review of effectiveness and cost-effectiveness
}

\author{
This article was published in the following Dove Press journal: \\ Clinical Ophthalmology \\ I4 August 2014 \\ Number of times this article has been viewed
}

\author{
Renata Linertová ${ }^{1,2}$ \\ Rodrigo Abreu-González ${ }^{3}$ \\ Lidia García-Pérez'1,2 \\ Marta Alonso-Plasencia ${ }^{3}$ \\ Luis Mateo Cordovés- \\ Dorta ${ }^{4}$ \\ José Augusto Abreu-Reyes ${ }^{4}$ \\ Pedro Serrano-Aguilar ${ }^{2,5}$ \\ 'Fundación Canaria de Investigación \\ y Salud (FUNCIS), Santa Cruz de \\ Tenerife, Spain; ${ }^{2}$ Red de Investigación \\ en Servicios Sanitarios en \\ Enfermedades Crónicas (REDISSEC), \\ Madrid, Spain; ${ }^{3}$ Ophthalmology \\ Service, University Hospital Ntra \\ Sra de La Candelaria, Santa Cruz \\ de Tenerife, Spain; ${ }^{4}$ Ophthalmology \\ Service, University Hospital of Canary \\ Islands, La Laguna, Spain; ${ }^{5} \mathrm{HTA}$ Unit, \\ Canary Health Service, Santa Cruz de \\ Tenerife, Spain
}

Correspondence: Renata Linertová Servicio de Evaluación, Servicio Canario de Investigación y Salud (FUNCIS), Camino Candelaria 44, I ${ }^{\text {P }}$ Planta, 38109 El Rosario, Santa Cruz de Tenerife, Spain Tel +34922684019

Email renata.linertova@sescs.es

\begin{abstract}
Postoperative endophthalmitis is one of the most serious potential complications of ocular lens surgery. Its incidence can be reduced by means of antibiotic prophylaxis. Although the prophylactic use of intracameral cefuroxime has been extended, other drugs, such as moxifloxacin, have arisen as alternatives. We performed a systematic literature review on the effectiveness and efficiency of intracameral cefuroxime and moxifloxacin for the prophylaxis of postoperative endophthalmitis after cataract surgery. Several bibliographic databases were searched up to October 2010 and were updated up to January 2013. Outcomes were the onset of endophthalmitis after surgery and the cost-effectiveness ratio of using both antibiotic prophylaxis alternatives. The following were included: a clinical trial reported in two papers, six observational studies, and an economic evaluation. All studies assessed cefuroxime compared with another antibiotic prophylaxis or no prophylaxis. The only randomized controlled trial performed by the European Society of Cataract and Refractive Surgery found that intracameral cefuroxime is significantly more effective than not using prophylaxis or the use of a topical antibiotic. The observational studies support these results. The economic evaluation compared different prophylaxis regimens and concluded that intracameral cefuroxime showed the best cost-effectiveness ratio. Both the observational studies and the economic evaluation have methodological limits that reduce their validity. This review confirmed that cefuroxime can prevent endophthalmitis after cataract surgery. Further randomized controlled trials, with large sample sizes, are required to compare different antibiotic prophylaxis regimens.
\end{abstract}

Keywords: cefuroxime, moxifloxacin, intracameral, systematic review, endophthalmitis, prophylaxis, antibiotics

\section{Introduction}

Postoperative endophthalmitis is one of the most feared complications of intraocular surgery. It may significantly compromise visual function and even the anatomical integrity of the eye.

The ethology of microorganisms infecting the eye during cataract surgery include the following: the patient's own ocular surface flora, infection stemming from contaminated surgical instruments, surgical complications, poor or delayed wound healing, and patients presenting preoperatively with blepharitis and inflammation or infection of the eyelids. The majority of bacteria causing endophthalmitis after cataract surgery in Western countries are Gram-positive microbes, described with varying frequency in reported series. Bacteria most commonly identified in endophthalmitis after cataract surgery in many Western countries may include coagulase-negative staphylococci 
(Staphylococcus epidermidis), Staphylococcus aureus (including methicillin-resistant $S$. aureus), $\beta$-hemolytic streptococci, and Enterococcus faecalis among Gram-positive organisms; Gram-negative rods, including Haemophilus influenzae; and Pseudomonas aeruginosa among Gramnegative microorganisms. ${ }^{2}$ Some of these microorganisms causing post-cataract surgery endophthalmitis could be multidrug resistant. ${ }^{3}$

The measures most commonly used to reduce the risk of postoperative endophthalmitis are preoperative use of iodized povidone and, more recently, the use of intracameral antibiotics on completion of surgery. ${ }^{4-7}$ The administration of antibiotics in the anterior chamber after surgery is theoretically the most direct method for prophylaxis. Although its use has already been reported previously, ${ }^{8-10}$ it has been recently disseminated in Europe as a result of the multicenter, prospective, randomized controlled trial performed by the European Society of Cataract and Refractive Surgeons (ESCRS $)^{6}$ using cefuroxime. In spite of the results of this pioneer study, we require further scientifically valid information on effectiveness, safety, and cost-effectiveness to guide us in decisions and potentially modify clinical practice in the quest for converging aims to improve health outcomes. A proposed intracameral alternative to cefuroxime is moxifloxacin, which offers a broader activity spectrum and a concentration-dependent action mechanism. ${ }^{11}$

The purpose of this systematic review is to evaluate consequences of this requirement and available scientific knowledge on the effectiveness and cost-effectiveness of using intracameral cefuroxime and moxifloxacin to reduce the incidence and complications associated with endophthalmitis after cataract surgery.

\section{Materials and methods used}

We performed a systematic review of existing scientific literature with results on the effectiveness and cost-effectiveness of intracameral moxifloxacin and cefuroxime for prophylaxis of postoperative endophthalmitis at the close of cataract surgery.

\section{Sources of information and search strategy}

Search strategies in the bibliographical databases MEDLINE and PreMEDLINE, EMBASE, CINAHL, CRD, and CENTRAL of the Cochrane Library (up to October 2010) were designed and implemented; the search was updated on MEDLINE and PreMEDLINE until January 2013. The main descriptors used in the bibliographical search were
Medical Subject Headings (MeSH) terms such as "cataract", "endophthalmitis", "Gram-positive bacterial infections", "antibiotic prophylaxis", "cefuroxime", or "moxifloxacin" (the full search strategy can be seen in Linertová et al). ${ }^{12}$ When carrying out the searches, no restrictions were applied by dates or by language.

\section{Selection criteria for studies}

Four reviewers selected in pairs those papers that could comply with the inclusion criteria from reading their titles and summaries. In case of doubt, a third reviewer assisted with the selection. The references selected were obtained and the full text read in order to finally decide whether or not to include or exclude the review. The selection criteria for the studies were the following.

\section{Types of participant}

Men and women of any age operated on for transparent lens or cataract were included. Those studies that included congenital cataract, secondary cataract, traumatic cataract, combined cataract surgery and glaucoma, or cataract and vitreoretinal surgery, and studies in which patients had been selected not only because of presenting lens pathology but also because of presenting comorbidity, were excluded.

\section{Types of procedure}

Prophylactic bolus injections of antibiotic solution (cefuroxime and/or moxifloxacin) were administered into the anterior chamber at the end of cataract surgery.

\section{Types of studies}

Clinical trials (randomized and nonrandomized) and observational studies such as cohort studies, case control studies, and cross-sectional studies, including the group of cases with number of cases greater than ten, were included. Economic evaluations were also included. Secondary studies, editorials, and letters to the editor were excluded. Other studies (clinical practice guides and systematic literature reviews) were considered only with the aim of identifying other primary studies that did not appear in the online search.

\section{Types of outcomes}

Effectiveness of prophylaxis of postoperative endophthalmitis was measured by: 1) onset of presumed endophthalmitis (only clinical) during the first 6 weeks after surgery; and 2 ) onset of confirmed endophthalmitis (polymerase chain reaction or positive cultures) during the first 6 weeks after surgery. The measurements of interest from the economic studies were cost-efficiency ratios and the incremental 
cost-efficiency ratio, or, failing this, the amount of resources used and the cost of each treatment.

\section{Data extraction and assessment of methodological quality}

Data extraction and quality assessment were performed by four reviewers and verified by the lead reviewer. An ad hoc data extraction sheet was designed for the data extraction. Data to extract were those related to identification of the paper (authors, date of publication, country where the study was performed, financing), the methodology (type of study, follow-up, type of prophylaxis, comparator, number of patients, patient selection criteria, end points assessed), and the study results (onset of presumed or confirmed endophthalmitis, cost, or cost-effectiveness).

The methodological quality of the studies included was assessed by means of Critical Appraisal Skills Programme instruments for critical reading of clinical trials, cohort studies, and case and control studies. ${ }^{13}$ We performed a narrative summary with results tabulated by subgroups based on study design.

\section{Results}

During the first search carried out in 2010, 1,000 references of published papers were located. Of these, 956 were excluded from the assessment of summaries or titles because of not complying with the inclusion criteria set out in the protocol. The full texts of the remaining 44 papers were reviewed, and it was found that 40 of these did not comply with the inclusion criteria, for which reason they were excluded from the review. From the review of bibliographical references of the primary studies included and a manual search, three additional references were located. Therefore, seven references were finally included in this phase: one clinical trial reported in two papers, ${ }^{6,14}$ four observational studies, ${ }^{8,15-17}$ and an economic evaluation. ${ }^{18}$

In the update (MEDLINE and PreMEDLINE between October 2010 and January 2013), 106 new references were identified, of which only five were reviewed as full text after initial review of the contents of titles and summaries, and two complied with this review's inclusion criteria. ${ }^{19,20}$ Therefore, nine references were included in this systematic review (Figure 1).

\section{Effectiveness}

All the studies included evaluated the effectiveness of intracameral cefuroxime compared with another kind of antibiotic prophylaxis or no prophylaxis. The economic evaluation
$1000+106$ identified

records without duplicates

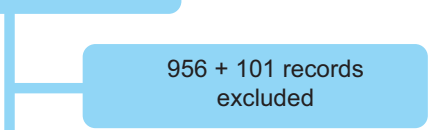

$44+5$ full-text articles assessed for eligibility

$40+3$ records excluded

$4+2$ studies included

+3 additional records by means of manual search

Figure I Flow diagram of study selection (original search plus update search).

also included topical and intracameral moxifloxacin as an alternative comparison. However, we did not find any paper on the use of intracameral moxifloxacin for the prophylaxis of endophthalmitis after cataract surgery (Table 1).

\section{Clinical trials}

The only prospective randomized and multicenter clinical trial on the prophylaxis of endophthalmitis with intracameral cefuroxime was performed by the ESCRS; this was subsequently published in two different papers: Barry et a ${ }^{14}$ gave preliminary information on the promising results of cefuroxime, and, subsequently, ESCRS ${ }^{6}$ published the final trial results. The trial was designed to recruit approximately 35,000 patients. However, it was discontinued approximately halfway through because of a recommendation by the ethics committee, whose opinion it was that it would not be ethical to continue not to use cefuroxime in the two arms of the trial. In the end, the sample for analysis by intention to treat consisted of 16,211 patients.

The rates of onset of confirmed postoperative endophthalmitis (by culture and/or polymerase chain reaction) in the two groups that did not receive cefuroxime were $0.247 \%$ (control group) and $0.173 \%$ (levofloxacin), compared with the ratios of $0.049 \%$ in the cefuroxime group and $0.025 \%$ in the group cefuroxime plus levofloxacin (Table 1). These numbers come from intention-to-treat analysis.

\section{Observational studies}

In addition, six observational studies on the prophylactic use of intracameral cefuroxime have been identified (Table 1). 


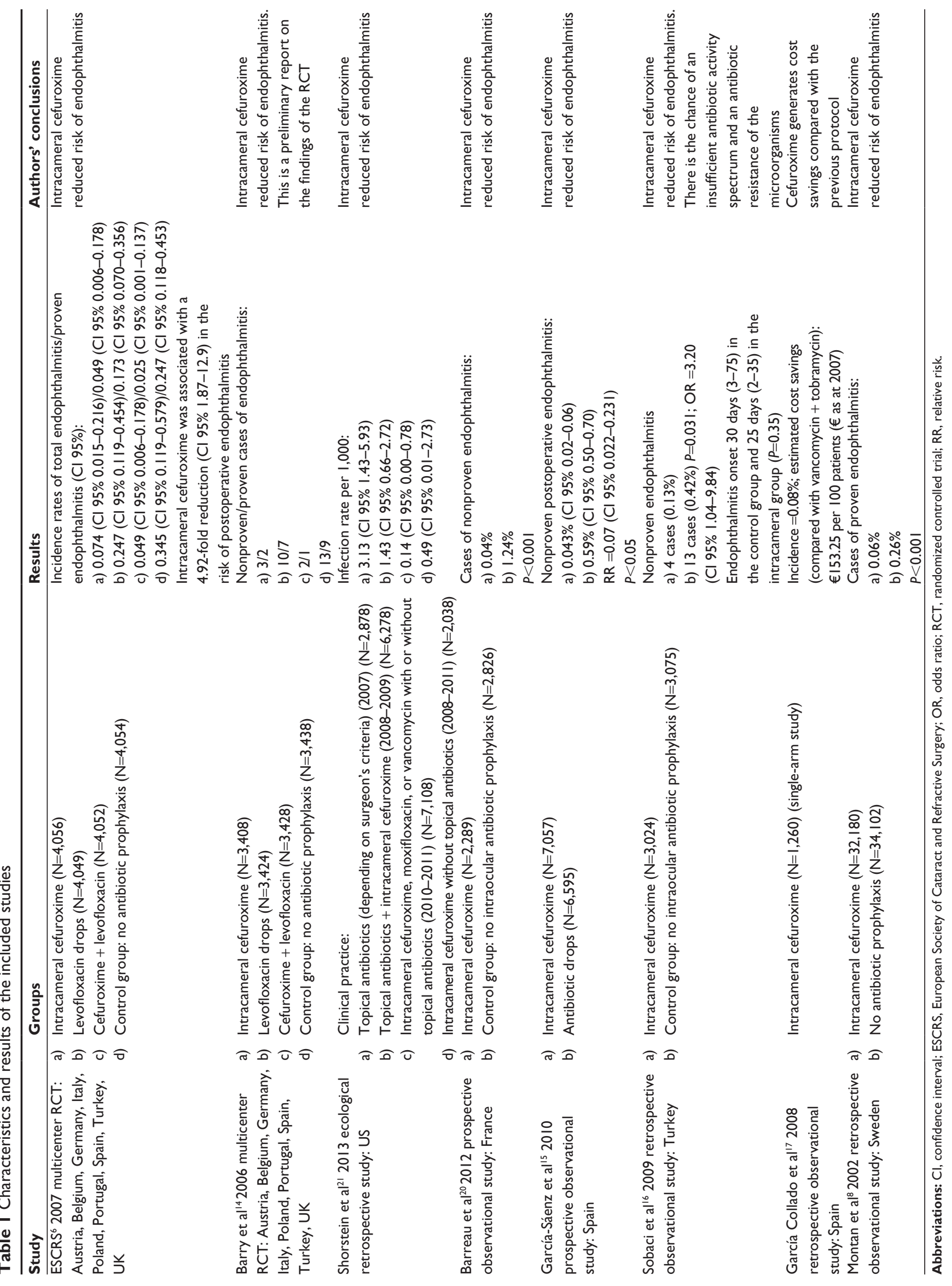


The most recent study, published in 2013, was performed in the US and involved an ecological study of 16,264 cataract procedures performed by 14 surgeons. ${ }^{19}$ The authors observed the changes on the incidence of endophthalmitis over 5 years (2007-2011) as the clinical practice of antibiotic prophylaxis changed; the prophylaxis policy was altered in September 2007 when intracameral cefuroxime was introduced. This change led to a reduction in the infection rate from 3.13 to 1.43 cases per 1,000 interventions. During 2010 and 2011 all patients were injected with cefuroxime, moxifloxacin, or vancomycin, and the rate went down to 0.14 cases per 1,000 (Table 1). Of the 19 cases of endophthalmitis, three received intracameral cefuroxime and one intracameral moxifloxacin; the remaining cases received only topical antibiotics. The incidence of endophthalmitis for each intracameral agent was not established, as the analysis would not have sufficient power to be able to draw conclusions on its comparative effectiveness. ${ }^{21}$ The study concluded that the use of intracameral antibiotics is associated with a reduction in the incidence of postoperative endophthalmitis after cataract surgery.

A study performed in France and published in $2012^{20}$ prospectively analyzed two cohorts based on a change in prophylaxis protocol. A total of 2,826 patients operated on between April 2003 and May 2006 did not receive intracameral cefuroxime, whereas 2,289 patients operated on between June 2006 and June 2008 did receive it. A total of 35 cases of endophthalmitis were observed in the group that did not receive cefuroxime $(1.24 \%)$ and one case in the cefuroxime group $(0.04 \%)(P<0.001)$. In multivariate analyses, the absence of cefuroxime was the only statistically significant risk factor for postoperative endophthalmitis after cataract surgery.

Another study with a similar design and a larger sample, performed in Spain and published in 2010, ${ }^{15}$ analyzed electronic clinical histories of a total of 13,652 patients operated on for cataract, and compared two cohorts based on a change in prophylaxis protocol. Patients operated on from October 2005 to December 2008 received intracameral cefuroxime according to the ESCRS trial recommendations. The control group comprised patients operated on from January 1999 to September 2005 who received topical antibiotics and/or steroids. The rate of onset of endophthalmitis was $0.59 \%$ in the period prior to October 2005, whereas during the subsequent period the rate was only $0.043 \%$, which led to a relative risk of 0.07 (range $0.022-0.231 ; P<0.05$ ) (Table 1). The study supported the conclusions of the clinical trial by ESCRS. ${ }^{6}$ Another Spanish study, ${ }^{17}$ which followed a retrospective observational design of just one cohort, analyzed 1,260 cataract procedures with intracameral cefuroxime. The prevalence of postoperative endophthalmitis was $0.08 \%$; only one case of postoperative endophthalmitis was recorded. In addition, the authors estimated cost savings due to the use of intracameral cefuroxime (see the Costs and cost-effectiveness section).

A retrospective observational study performed in Turkey in $2009^{16}$ compared a cohort of 3,024 cataract procedures not receiving intracameral antibiotic prophylaxis with a cohort of 3,075 cataract procedures in which intracameral cefuroxime was applied. The onset of endophthalmitis was $0.42 \%$ in the control group (period up to 2004), compared with $0.13 \%$ in the group that received cefuroxime (period between 2004 and 2007) $(P=0.031)$ (Table 1). A study performed in Sweden in $2002^{8}$ with a similar design included two cohorts. The first one included 34,102 participants operated on for cataract between 1990 and 1995 without intracameral prophylaxis. The second group included 32,180 patients operated on between 1996 and 2000, during which intracameral cefuroxime was administered. The rate of incidence of endophthalmitis was calculated as $0.06 \%$ in the intervention group and $0.26 \%$ in the control group $(P<0.001)$.

\section{Costs and cost-effectiveness}

The only full economic evaluation of endophthalmitis prophylaxis dates back to 2009 . This was performed in the US and was not funded by the pharmaceutical industry. ${ }^{18}$ Its aim was to assess the cost-effectiveness of intracameral cefuroxime as antibiotic prophylaxis for postoperative endophthalmitis after cataract surgery from the point of view of society, and to determine the cost-effectiveness threshold for alternative antibiotics to be compared with intracameral cefuroxime. For the purpose of our aims, we should note that among the alternatives both topical and intracameral administration of moxifloxacin were also assessed.

The results (Table 2) reveal that the use of intracameral cefuroxime saves costs, considering the costs avoided in cases of endophthalmitis, prevented by using the antibiotic. For moxifloxacin's cost-effectiveness to be compared with the cost-effectiveness threshold of cefuroxime, moxifloxacin would have to be almost five times more effective than cefuroxime (prevent five times more cases). However, we have to be careful when interpreting these results, because of the limitations that could not be resolved by this study. First, the analysis parameters for costs and effectiveness and prevalence were obtained from different sources of literature, and in some cases the authors had to make some assumptions. 
Table 2 Results of the economic evaluation

\begin{tabular}{llll}
\hline Antibiotics & Cohort net cost (in US\$ millions) & Cost-effectiveness ratio & Threshold effectiveness ratio* \\
\hline Intracameral cefuroxime & 0.48 & Saving & No results available \\
Topical sulfacetamide & 0.71 & Saving & $0.8 \mathrm{I}$ \\
Subconjunctival gentamicin & 0.64 & Saving & 1.04 \\
Subconjunctival cefazolin & 0.58 & Saving & 1.26 \\
Topical polymixin/trimethoprim & 0.30 & 1,211 & 4.36 \\
Combination** & 0.40 & 1,976 & 4.11 \\
Intracameral moxifloxacin & 0.44 & 1,800 & 4.87 \\
Topical ciprofloxacin & 1.55 & 6,288 & 8.79 \\
Topical ofloxacin & 2.44 & 9,867 & 11.90 \\
Topical moxifloxacin & 4.56 & 18,474 & 19.40 \\
Topical gatifloxacin & 4.82 & 19,527 & 20.32 \\
\hline
\end{tabular}

Notes: *Threshold effectiveness ratio for each antibiotic to achieve the same cost-effectiveness ratio as intracameral cefuroxime. $* *$ Combination of intracameral cefuroxime, subconjunctival cefazolin, subconjunctival gentamicin, and topical sulfacetamide. Data from Sharifi et al. ${ }^{16}$

Secondly, the unitary costs are difficult to transfer between countries. Finally, this analysis does not indicate whether a prophylaxis regimen is clinically better than another, as there are insufficient data for moxifloxacin regimens.

Noteworthy in this section is a cost study performed in Spain that was included in this review. ${ }^{17}$ The authors attempted to quantify the financial impact of the incorporation of cefuroxime into habitual cataract surgery practice, compared with the previous prophylaxis protocol that included topical vancomycin and tobramycin. The cost analysis included only direct costs of material. It was estimated that the new protocol (intracameral cefuroxime) could provide a financial saving of $€ 153.25$ for every 100 patients ( $€$ from 2007). We have to bear in mind that this study is not a full economic evaluation but rather a cost estimate under very specific conditions.

\section{Methodological quality of the papers included}

The quality of the studies included was assessed by the Critical Appraisal Skills Programme instrument method for assessment of the validity of clinical trials and cohort studies. ${ }^{13}$

The ESCRS clinical trial ${ }^{6,14}$ complied with all good-quality methodological criteria (Table 2); patients were randomly assigned to treatments and all the relevant characteristics of the groups at onset were similar.

Four observational studies included in this review compared an intervention cohort with another historic cohort, which entails the risk of possible uncontrollable bias such as population changes or improvements in surgical conditions; $, 8,15,16,20$ one study reported just one cohort without comparison ${ }^{16}$ (Table 1). Because of its design, the ecological study cannot compare strictly defined cohorts, as it observes course in clinical practice and serves to generate hypotheses. ${ }^{19}$
Therefore, the validity and quality of these results are limited by the design of the studies.

\section{Discussion}

Postoperative endophthalmitis is the most devastating complication of cataract surgery, though its incidence is fortunately very low because of technical and pharmacologic improvements that have contributed both to reducing the aggressivity of surgery and procedure times and to improving defense capacity with asepsis and antibiotherapy. ${ }^{11,22,23}$ These technological improvements, together with the observation of very low rates of incidence of endophthalmitis, might be contributing to the observed increasing use of simultaneous bilateral cataract surgery for specific indications and selected patients. ${ }^{24,25}$ Nonetheless, we should consider that this highfrequency surgery is performed on millions of people every year globally, ${ }^{23,24}$ for which reason we need to maximize the guarantees of effectiveness and safety from the point of view of patients, and cost-effectiveness from the point of view of the health system.

Recent progress in the use of intracameral antibiotherapy appears to contribute to reducing the risk of endophthalmitis for cataract surgery. ${ }^{19,26,27}$ Although the use of antibiotics can be extended preoperatively, intraoperatively, and postoperatively, there is only sufficient scientific evidence to justify the use of intracameral cefuroxime at the end of the procedure. ${ }^{6,14,28}$ The value of using other antibiotics by this route, seeking possible advantages over cefuroxime, has been suggested, although this has not been proven. ${ }^{11}$

This systematic review gives favorable results for the prophylactic use of intracameral cefuroxime at the end of cataract surgery. Regarding the use of moxifloxacin as an alternative to cefuroxime for this same indication, there is no valid information that confirms its effectiveness or 
cost-effectiveness, for which reason it is regarded as a promising antibiotic that requires further investigation.

We have verified that the effectiveness of cefuroxime is revealed in several studies with large samples. The study with most scientific weight is the clinical trial performed by ESCRS $^{6,14}$ in which intracameral cefuroxime revealed significant benefits compared with no prophylaxis or topical antibiotic (levofloxacin). Results were clinically relevant and may be generalized to the Spanish population, as the study included patients from nine European countries, including Spain. The most recent study by Shorstein et al ${ }^{19}$ with a similar sample, confirms this conclusion by the ESCRS and adds that the reduction of endophthalmitis with intracameral antibiotics was regardless of the use of topical antibiotics such as gatifloxacin, that intracameral moxifloxacin was useful in patients allergic to penicillin, and that intracameral antibiotics are especially useful in cases of rupture of the posterior capsule. ${ }^{29}$

Compared with cefuroxime, moxifloxacin offers a broader activity spectrum and a concentration-dependent action mechanism that could be advantageous for intracameral administration. ${ }^{11}$ However, our systematic review has not found valid clinical studies that evaluate its effectiveness, making it impossible to draw conclusions; there are studies on the safety of its use by this route. ${ }^{30}$ Another theoretical advantage is that moxifloxacin is marketed as self-preserved eye drops that could be used directly by the intracameral route, although this form of administration does not appear in the technical specifications (VIGAMOX ${ }^{\circledR}$; Alcon Laboratories, Inc., Fort Worth, TX, USA). ${ }^{31}$

The main limitation of our review was the low number of papers found that complied with the selection criteria. In spite of cataract surgery being so frequent, we found only one randomized clinical trial with the use of intracameral cefuroxime, ${ }^{6,14}$ no reference to endophthalmitis prophylaxis with the use of intracameral moxifloxacin as first-line drug, and only one economic evaluation. ${ }^{18}$ To the best of our knowledge, our work is the first systematic literature review on intracameral prophylaxis for endophthalmitis after lens surgery. However, it is true that the lack of literature in this regard does not enable us to perform a quantitative summary of the data or set out categorical recommendations.

In 2007 an online survey was carried out on the 4,000 members of the ASCRS to assess the impact produced by the ESCRS clinical trial. ${ }^{32}$ Of the 1,312 responses received, a minority $(30 \%)$ used intracameral antibiotics during cataract surgery. The survey authors concluded that the ESCRS clinical trial had not modified the usual clinical practice of $77 \%$ of those surveyed, in spite of the fact that $82 \%$ would like to use intracameral antibiotics.

One of the obstacles for generalized use of cefuroxime for prophylactic purposes was not currently having a specific commercial preparation at the necessary concentration $(0.1 \mathrm{mg} / \mathrm{mL})$. However, in 2012 a specific preparation called Aprokam $^{\circledR}$ (Laboratoires Théa, Clermont-Ferrand, France) $)^{33}$ had already appeared in Europe in 2012 and will appear in the remaining European countries in 2013.

It is interesting to ascertain the effectiveness and costeffectiveness of other possible interventions that are alternatives to intracameral cefuroxime. The possible alternatives to cefuroxime, such as moxifloxacin or other antibiotics, should provide information from direct comparisons on the effectiveness to reduce the incidence of endophthalmitis, and other less relevant infection-related complications, on possible potentially associated adverse events, and cost-effectiveness, by means of comparative studies compared with active comparator (cefuroxime) and not compared with only placebo.

\section{Acknowledgment}

Funded by the Quality Plan for the National Health System, Health Ministry of Spain.

\section{Disclosure}

The authors report no conflicts of interest in this work.

\section{References}

1. ESCRS guidelines on prevention, investigation and management of post-operative endophthalmitis [webpage on the Internet]. The European Society for Cataract and Refractive Surgeons; 2007. Available from: http://www.chulaophthalmology.org/userfiles/download/ ESCRS_Guidelines_2007.pdf. Accessed February 1, 2013.

2. ESCRS guidelines on prevention and treatment of endophthalmitis following cataract surgery [webpage on the Internet]. The European Society for Cataract and Refractive Surgeons; 2013. Available from: http:// www.escrs.org/endophthalmitis/guidelines/ENGLISH.pdf. Accessed January 15, 2014.

3. Maltezou HC, Pappa O, Nikolopoulos G, et al. Post-cataract surgery endophthalmitis outbreak caused by multidrug-resistant Pseudomonas aeruginosa. Am J Infect Control. 2012;40(1):75-77.

4. Fintelmann RE, Naseri A. Prophylaxis of postoperative endophthalmitis following cataract surgery. Drugs. 2010;70(11):1395-1409.

5. Speaker MG, Menikoff JA. Prophylaxis of endophthalmitis with topical povidone-iodine. Ophthalmology. 1991;98:1769-1775.

6. ESCRS Endophthalmitis Study Group. Prophylaxis of postoperative endophthalmitis following cataract surgery: results of the ESCRS multicenter study and identification of risk factors. J Cataract Refract Surg. 2007;3:978-988.

7. Wu PC, Li M, Chang SJ. Risk of endophthalmitis after cataract surgery using different protocols for povidone-iodine preoperative disinfection. J Ocul Pharmacol Ther. 2006;22(1):54-61.

8. Montan PG, Wejde G, Koranyi G, Rylander M. Prophylactic intracameral cefuroxime. Efficacy in preventing endophthalmitis after cataract surgery. J Cataract Refract Surg. 2002;28:977-981. 
9. Wejde G, Montan P, Lundström M, Stenevi U, Thorburn W. Endophthalmitis following cataract surgery in Sweden: national prospective survey 1999-2001. Acta Ophthalmol Scand. 2005;83:7-10.

10. Gimbel HV, Sun R, DeBrof BM. Prophylactic intracameral antibiotics during cataract surgery: the incidence of endophthalmitis and corneal endothelial cell loss. Eur J Implant Ref Surg. 1994;6:280-285.

11. O'Brien TP, Arshinoff SA, Mah FS. Perspectives on antibiotics for postoperative endophthalmitis prophylaxis: potential role of moxifloxacin. J Cataract Refract Surg. 2007;33:1790-1800.

12. Linertová R, Abreu González R, García-Pérez L, Alonso Plasencia M. Profilaxis de la endoftalmitis postquirúrgica en cirugía del cristalino: cefuroxima y moxifloxacino intracamerular. Plan de Calidad para el Sistema Nacional de Salud del Ministerio de Sanidad y Política Social e Igualdad [Prophylaxis of postoperative endophthalmitis in the lens surgery: Intracameral cefuroxime and moxifloxacine]. Servicio de Evaluación del Servicio Canario de la Salud; 2011. Informes de Evaluación de Tecnologías Sanitarias: SESCS No 2010/04. Spanish.

13. CASP España. Programa de Lectura Crítica CASPe. Entendiendo la evidencia sobre la eficacia clínica. 11 preguntas para ayudarte a entender un estudio de cohortes [Critical Appraisal Skills Programme Spain. Understanding clinical efficacy evidence. 11 questions to help you understand a cohort study]. Available at: http://www.redcaspe.org/ drupal/?q=node/29. Accessed July 7, 2014. Spanish.

14. Barry P, Seal DV, Gettinby G, Lees F, Peterson M, Revie CW; ESCRS Endophthalmitis Study Group. ESCRS study of prophylaxis of postoperative endophthalmitis after cataract surgery: preliminary report of principal results from a European multicenter study. J Cataract Refract Surg. 2006;32:407-410

15. García-Sáenz MC, Arias-Puente A, Rodríguez-Caravaca G, Bañuelos JB. Effectiveness of intracameral cefuroxime in preventing endophthalmitis after cataract surgery. Ten-year comparative study. J Cataract Refract Surg. 2010;36:203-207.

16. Sobaci G, Uysal Y, Mutlu FM, Bayer A, Gungor R, Karagul S. Prophylactic usage of intracameral cefuroxime in the prevention of postoperative endophthalmitis. Klin Monbl Augenheilkd. 2009;5:265-267.

17. García Collado C, Morillo Verdugo R, Montes Escalante I, Muñoz Muñoz N, Artacho Criado S, Dorantes Calderón B. Cefuroxima $1 \mathrm{mg} / 0,1 \mathrm{~mL}$ en cámara anterior para profilaxis de endoftalmitis postquirúrgica en cirugía de cataratas [Intracameral $1 \mathrm{mg} / 0,1 \mathrm{~mL}$ cefuroxime in postoperative endophthalmitis prophylaxis in cataract surgery]. Aten Farm. 2008;10(1):44-47. Spanish.

18. Sharifi E, Porco TC, Naseri A. Cost-effectiveness analysis of intracameral cefuroxime use for prophylaxis of endophthalmitis after cataract surgery. Ophthalmology. 2009;116:1887-1896.

19. Shorstein NH, Winthrop KL, Herrinton LJ. Decreased postoperative endophthalmitis rate after institution of intracameral antibiotics in a Northern California eye department. J Cataract Refract Surg. 2013;39(1):8-14.
20. Barreau G, Mounier M, Marin B, Adenis JP, Robert PY. Intracameral cefuroxime injection at the end of cataract surgery to reduce the incidence of endophthalmitis: French study. J Cataract Refract Surg. 2012;38(8):1370-1375.

21. Shorstein N, Winthrop K, Herrinton L. Reply: intracameral antibiotics and endophthalmitis incidence. J Cataract Refract Surg. 2013;39(2):313

22. Fintelmann RE, Naseri A. Prophylaxis of postoperative endophthalmitis following cataract surgery. Drugs. 2010;70(11):1395-1409.

23. Behndig A, Montan P, Stenevi U, Kugelberg M, Lundström M. One million cataract surgeries: Swedish National Cataract Register 1992-2009. J Cataract Refract Surg. 2011;37:1539-1545.

24. Arshinoff SA, Bastianelli PA. Incidence of postoperative endophthalmitis after immediate sequential bilateral cataract surgery. J Cataract Refract Surg. 2011;37(12):2105-2114.

25. Sarikkola AU, Uusitalo RJ, Hellstedt T, et al. Simultaneous bilateral versus sequential bilateral cataract surgery: Helsinki Simultaneous Bilateral Cataract Surgery Study Report 1. J Cataract Refract Surg. 2011;37(6):992-1002.

26. Serrano-Aguilar P, Ramallo-Fariña Y, Cabrera-Hernández JM, et al. Immediately sequential versus delayed sequential bilateral cataract surgery: safety and effectiveness. J Cataract Refract Surg. 2012;38(10):1734-1742.

27. Miller JJ, Scott IU, Flynn Jr HW. Acute-onset endophthalmitis after cataract surgery (2000-2004): incidence, clinical settings, and visual acuity outcomes after treatment. Am J Ophthalmol. 2005;139(6):983-987.

28. Ang GS, Barras CW. Prophylaxis against infection in cataract surgery: a Surrey of Soutine practice. Eur J Ophthalmol. 2006;16:394-400.

29. Barry P. Intracameral antibiotic prophylaxis: American paper mirrors European experience. J Cataract Refract Surg. 2013;39(1):2-3.

30. Lane SS, Osher RH, Masket S, Belani S. Evaluation of the safety of prophylactic intracameral moxifloxacin in cataract surgery. J Cataract Refract Surg. 2008;34:1451-1459.

31. VIGAMOX ${ }^{\circledR}$ (moxifloxacin hydrochloride ophthalmic solution) Highlights of prescribing information [webpage on the internet]. TX: Alcon Laboratories, Inc.; 2011 [Updated July, 2011]. Available from: http:// ecatalog.alcon.com/pi/Vigamox_us_en.pdf. Accessed May 7, 2014.

32. Chang DF, Braga-Mele R, Mamalis N, et al. Prophylaxis of postoperative endophthalmitis after cataract surgery; results of the 2007 ASCRS member survey; the ASCRS Cataract Clinical Committee. J Cataract Refract Surg. 2007;33:1801-1805.

33. Protocole d'utilisation therapeutique et de recueil d'informations. Aprokam $50 \mathrm{mg}$, poudre pour solution injectable (céfuroxime) [Therapeutic protocol use and information collection. Aprokam $50 \mathrm{mg}$ powder for solution for injection (cefuroxime)]. Available from: http:// ansm.sante.fr/var/ansm_site/storage/original/application/90a18ec1748 dba109c2b6efe77ec6775.pdf. Accessed July 7, 2014. French.
Clinical Ophthalmology

\section{Publish your work in this journal}

Clinical Ophthalmology is an international, peer-reviewed journal covering all subspecialties within ophthalmology. Key topics include: Optometry; Visual science; Pharmacology and drug therapy in eye diseases; Basic Sciences; Primary and Secondary eye care; Patient Safety and Quality of Care Improvements. This journal is indexed on Submit your manuscript here: http://www.dovepress.com/clinical-ophthalmology-journal
Dovepress

PubMed Central and CAS, and is the official journal of The Society of Clinical Ophthalmology (SCO). The manuscript management system is completely online and includes a very quick and fair peer-review system, which is all easy to use. Visit http://www.dovepress.com/ testimonials.php to read real quotes from published authors. 